[6] A. Martineau, Sur une propriété caractéristique d'un produit de droites, Arch. Math. 11 (1960), p. $423-426$.

[7] A. Pietsch, Homomorphismen in lokalkonvexen Vektorräumen, Math. Nachr. 22 (1960), p. 162-174.

[8] V. Ptak, Completeness and the open mapping theorem, Bull. Soc. Math. France 86 (1958), p. $41-74$

[9] H. H. Schaefer, Topological vector spaces, 1966.

\title{
Equivalent nuclear systems
}

by

Ed. DUBINSKY (Hamilton, Ont.)

In this paper we introduce the notion of nuclear system as a means of constructing nuclear Fréchet spaces whose topologies are defineđ by a family of seminorms which are actually norms. We then show that all such spaces are obtained by this construction. The main result (Theorem 2) is an "intrinsic" characterization of when two nuclear systems are equivalent, that is when the spaces which they construct are isomorphic. This result is then applied to the basis problem for nuclear Fréchet spaces. Finally some examples and open questions are listed.

This method of constructing nuclear Fréchet spaces gives rise to examples which have not previously been discussed as well as providing a new way of studying the familiar spaces. These examples will be discussed in detail in a forthcoming paper.

Let $A_{k}: l_{2} \rightarrow l_{2}, k=1,2, \ldots$, be a sequence of nuclear maps and define the associated space;

$$
\hat{E}=\hat{E}\left\{\left(A_{k}\right)\right\}=\left\{\left(x_{k}\right)_{k}: x_{k} \in l_{2}, x_{k}=A_{k} x_{k+1}, k=1,2, \ldots\right\} .
$$

Thus $\hat{\boldsymbol{E}}$ is a subspace of the countable product of copies of $l_{2}$, and we may equip $\hat{E}$ with the topology induced by the usual product topology. Let $P_{k}: \hat{E} \rightarrow l_{2}$ by $P_{k}\left(\left(x_{k}\right)_{k}\right)=x_{k}$. We call $\left(A_{k}\right)_{k}$ a nuclear system if

(i) each $A_{\%}$ has dense range

(ii) each $P_{k}$ is injective.

THEOREM 1. The associated space of nuclear system is a nuclear Fréchet space with a fundamental sequence of seminorms which are norms; and, conversely, every such space is the associated space of a nuclear system (up to isomorphism).

Proof. Clearly, $\hat{E}\left\{\left(A_{k}\right)\right\}$ is nothing more than the projective limit of the sequence of maps, $\left(A_{k}\right)_{k}$ and hence it is a Fréchet space. Evidently, a fundamental system of neighborhoods of 0 is given by the sets

$$
V_{n}=\left\{\left(x_{k}\right) \in \hat{\boldsymbol{E}}:\left\|x_{k}\right\| \leqslant \frac{1}{n}, k=1,2, \ldots, n\right\}, \quad n=1,2, \ldots
$$


and it follows immediately from (ii) that the gage of each $V_{n}$ is a norm. To see that $\hat{E}$ is nuclear, set

$$
\|x\|_{n}=n \text { gage }\left(\nabla_{n}\right)=\max \left\{\left\|x_{1}\right\|, \ldots,\left\|x_{n}\right\|\right\}, \quad x=\left(x_{k}\right) \in \hat{E}, n=1,2, \ldots
$$

Then it suffices to show that any $\|\cdot\|_{n+1}$-summable sequence, $\left(x^{p}\right)_{2}$ in $\hat{E}$, is $\|\cdot\|_{n}$-absolutely summable.

Now if $\sigma$ runs through the collection of finite sets of positive integers, ordered by inclusion, and $S_{\sigma}=\sum_{v \in \sigma} x^{\nu}$, then the net $\left(S_{\sigma}\right)_{\sigma}$ is $\|\cdot\|_{n+1}$ - Cauchy. Hence for each $k \leqslant n+1$, the net $\left(P_{k}\left(S_{\sigma}\right)\right)_{\sigma}$ is Cauchy. In other words, if $x^{\prime \prime}=\left(x_{k}^{p}\right)_{k}$, then for each $k \leqslant n+1,\left(x_{k}^{y}\right)_{y}$ is a summable family in $l_{2}$ so $\left(A_{k} x_{k}^{v}\right)_{\nu}$ is absolutely summable for $k \leqslant n+1$, so $\left(x_{k}^{v}\right)_{v}$ is absolutely summable for each $k \leqslant n$, so $\left(x^{y}\right)_{\nu}$ is $\|\cdot\|_{h}$-absolutely summable.

For the converse let $E$ be given. Since $E$ is nuclear Fréchet, it is separable, so if $V$ is a barreled neighborhood of 0 , then $E_{V}$ is also separable. Thus we can choose a decreasing fundamental sequence of neighborhoods of $0,\left(\nabla_{k}\right)_{k}$, such that

$1^{\circ}$ the gage of each $\nabla_{k}$ is a norm,

$2^{\circ}$ each $\hat{E}_{V_{k}}$ is a separable Hilbert space,

$3^{\circ}$ the canonical map $\eta_{k}: \hat{E}_{V_{k+1}} \rightarrow \hat{E}_{V_{k}}$ is nuclear for $k=1,2, \ldots$

Then if $\theta_{k}: \hat{E}_{V_{k}} \rightarrow l_{2}$ is an isometry and $A_{k}=\theta_{k} \eta_{k} \theta_{k+1}^{-1}$, it is easy to check that $\left(A_{k}\right)_{k}$ is a nuclear system. Finally, it is well known (and straightforward to show) from the theory of projective limits that $E$ is isomorphic to $\hat{E}\left\{\left(\dot{A}_{k}\right)\right\}$.

This completes the proof of the theorem.

It is easy to see that in the definition of nuclear system, condition (ii) is satisfied if each $A_{k}$ is injective. If this is so, we shall say that the nuclear system is injective. This is not always the case, in fact we now give an example of a nuclear. system in which no $A_{k}$ is injective.

Let $e^{n}, n=1,2, \ldots$, be the usual basis for $l_{2}$ and define $A: l_{2} \rightarrow l_{2}$ by

$$
A e^{n}=\left\{\begin{array}{cc}
\frac{1}{4} e_{1} & \text { if } n=1, \\
\frac{1}{(n+1)^{2}} e^{n}-\frac{1}{(n-1)^{2}} e^{n-1} & \text { if } n>1
\end{array}\right.
$$

It is easy to see that $A$ is nuclear and also $e^{n} \in A\left(l_{2}\right)$ for $n=1,2, \ldots$, so $A$ has dense range. Thus if we take $A_{k}=A$ for $k=1,2, \ldots$, then we need only check condition (ii). In fact, we first observe that the kernel of $A$ is the subspace of $l_{2}$ generated by the vector: $x^{0}=\left(1 / n^{2}\right)_{n}$. Thus, if $\left(x_{k}\right) \in \hat{E}\left\{\left(A_{k}\right)\right\}$ and $x_{k}=0$ for some $k$, then $A x_{k+1}=0$ so we have a scalar $\lambda \neq 0$ with $x_{k+1}=\lambda x^{0}$. Hence

$$
x^{0}=A\left(\frac{1}{\lambda} \dot{x}_{k+2}\right) \in A\left(l_{2}\right) .
$$

But suppose that $x^{0}=A x, x=\left(x_{n}\right) \epsilon l_{2}$. Then by definition of $A$,

$$
\frac{x_{n}}{(n+1)^{2}}-\frac{x_{n+1}}{n^{2}}=\frac{1}{n^{2}}, \quad n=1,2, \ldots
$$

so

$$
x_{n}-\left(\frac{n+1}{n}\right)^{2} x_{n+1}=\left(\frac{n+1}{n}\right)^{2}, \quad n=1,2, \ldots
$$

Passing to the limit and using the fact that $\lim x_{n}=0$, we obtain

$$
0=\lim _{n} x_{n}=1 \text {, }
$$

which is a contradiction. Thus each $P_{k}$ is injective. On the other hand each $A_{k}=A$ has a non-trivial kernel and the desired example has been obtained.

It is natural to ask if every nuclear Fréchet space with a fundamental sequence of norms is the associated space of an injective nuclear system. To study this question we define two nuclear systems to be equivalent if their associated spaces are isomorphic. The main result of this paper will be to give an "intrinsic" characterization of equivalence and apply this to the basis problem for Fréchet nuclear spaces. To do this we must first show that the range of each $P_{k}$ is dense.

LEMTMa. Let $B_{k}: E_{k} \rightarrow E_{k+1}, k=1,2, \ldots$, be a sequence of injective linear continuous maps of reflexive Banach spaces. Then the inductive limit of these maps is Hausdorff.

Proof. This result is well known. For a proof see [1].

We now wish to make use of the duality between inductive and projective limits. To do this precisely, some notation is necessary. Let $F_{k}$ be the strong dual of $E_{k}$ and $A_{k}: F_{k+1} \rightarrow F_{k}$ the adjoint of $B_{k}$. Then by reflexivity, $B_{k}$ is the strong dual of $F_{k}$ and $B_{k}$ is the adjoint of $A_{k}$. Let $E$ be the inductive limit of $\left(B_{k}\right)$ and $F$ the projective limit of $\left(A_{k}\right)$. Then the situation is schematized in the diagrams
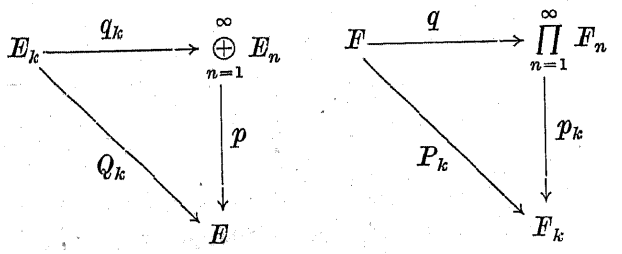
where the injections $q, q_{k}, Q_{k}$ and projections $p, p_{k}, Q_{k}$ have the usual meanings. The well known fact which we wish to use is that $F$ is the dual of $E$ in the sense that all elements of $E^{\prime}$ are obtained uniquely by taking. elements $u=\left(u_{n}\right)_{n}$ in $F$ and defining a linear functional on elements $x=\left(x_{n}\right)$ in $E$ by the relation,

$$
\langle x, u\rangle=\sum_{n=1}^{\infty}\left\langle x_{n}, u_{n}\right\rangle
$$

where this sum is finite because $x$ is finitely non-zero. With this notation, we may prove the

Proposition. Let $A_{k}: F_{k+1} \rightarrow F_{k}$ be a sequence of linear continuous maps of reflexive Banach spaces such that each $A_{k}\left(F_{k+1}\right)$ is dense in $F_{k}$ and let $F$ be the projective limit. Then for each $k, P_{k}\left(F^{\prime}\right)$ is dense in $F_{k}$.

Proof. In the notation described above, the injectivity of $B_{k}$ follows from the density of $A_{k}\left(F_{k+1}\right)$. Hence by the lemma, $E$ is Hausdorff. Now fix $k$ and suppose that $u \in E_{k}=F_{k}^{\prime}$ such that for each $x \in F^{\prime}$ we have $\left\langle P_{k} x, u\right\rangle=0$. Then,

$$
0=\left\langle P_{k} x, u\right\rangle=\left\langle p_{k} \circ q x, u\right\rangle=\left\langle x, p \circ q_{k} u\right\rangle=\left\langle x, Q_{k} u\right\rangle .
$$

Since this is true for all $x \in F$, it follows that $Q_{k} u=0$, and since $Q_{k}$ is injective (because $B_{k}$ is) we conclude that $u=0$. This shows that $P_{k}(F)$ is dense in $F_{k}$.

CoROLlaRY. If $\left(A_{k}\right)_{k}$ is a nuclear system, then each $P_{k}$ has dense range. Proof. The conditions of the proposition are obviously satisfied. THEOREM 2. Two nuclear systems $\left(A_{k}\right),\left(\tilde{A}_{k}\right)$ are equivalent if and only if there is a subsequence $\left(n_{k}\right)_{k}$ of indices and continuous linear maps $f_{k}: l_{2} \rightarrow l_{2}, k=1,2, \ldots$, such that
(i) $A_{k} f_{k+1}=f_{k} \tilde{A}_{n_{k}} \ldots \tilde{A}_{n_{k+1}-1}, k=1,2, \ldots$,
(ii) each $f_{k} \tilde{P}_{n_{k_{n}}}$ is injective,
(iii) $f_{k} \tilde{P}_{n_{k}}\left(\hat{E}\left\{\left(\tilde{A}_{k}\right)\right\}\right) \supset P_{k}\left(\hat{E}\left\{\left(A_{k}\right)\right\}\right), k=1,2, \ldots$
If $\left(A_{k}\right)$ is injective, then (ii) can be replaced by
(ii)'. $f_{1} \tilde{P}_{n_{1}}$ is injective

and (iii) can be replaced by

(iii) $f_{1} \tilde{P}_{n_{1}}\left(\hat{E}\left\{\left(\tilde{A}_{k}\right)\right\}\right) \supset P_{1}\left(\hat{E}\left\{\left(A_{k}\right)\right\}\right)$

or

(iii) $f_{1}\left(\bigcap_{k=1}^{\infty} \tilde{A}_{n_{1}} \ldots \tilde{A}_{n_{k-1}}\left(l_{2}\right)\right) \supset \bigcap_{k=1}^{\infty} A_{1} \ldots A_{k}\left(l_{2}\right)$.

Pro of. For convenience, we shall write $\hat{E}=\hat{E}\left\{\left(A_{k}\right)\right\}$ and $\hat{F}=\hat{E}\left\{\left(\tilde{A_{k}}\right)\right\}$. First suppose that the maps $f_{k}$ exist. For each $x \in \hat{F}$ and $k=1,2, \ldots$, define $y_{k}=f_{k} \tilde{P}_{n_{l c}}(x)$. Then

$$
A_{k}\left(y_{k+1}\right)=A_{k} f_{k+1} \tilde{P}_{n_{k+1}}(x)=f_{k} \tilde{A}_{n_{k}} \ldots \tilde{A}_{n_{k+1}-1} P_{n_{k+1}}(x)=f_{k} P_{n_{k}}(x)=y_{k} \text {. }
$$

Hence $\left(y_{k}\right) \epsilon \hat{E}$, so we can define $f: \hat{F} \rightarrow \hat{E}$ by

$$
P_{k e} f(x)=f_{k} \tilde{P}_{n_{k}}(x), \quad k=1,2, \ldots
$$

Clearly, $f$ is linear and continuous. Suppose $x \in \hat{F}$ and $f(x)=0$. Then each $f_{k} \tilde{P}_{n_{k}}(x)=0$, so by (ii), $x=0$. Thus $f$ is injective. Now let $y \in \hat{E}$. For each $k$, it follows from (iii) that there exists $x^{k} \in \tilde{F}$ with $P_{k}(y)=f_{k} \tilde{P}_{n_{k}}\left(x^{k}\right)$. But

$$
\begin{aligned}
f_{k} \tilde{P}_{n_{k}}\left(x^{k+1}\right) & =A_{k} f_{k+1} \tilde{P}_{n_{k+1}}\left(x^{k+1}\right)=A_{k} P_{k+1}(y) \\
& =P_{k}(y)=f_{k} \tilde{P}_{n_{k}}\left(x^{k}\right) .
\end{aligned}
$$

Hence by (ii) we have $x^{k}=x^{k+1}$ so $f\left(x^{1}\right)=y$ and $f$ is onto. Therefore by the open mapping theorem, $f$ is an isomorphism.

Conversely, suppose we have an isomorphism $f: \hat{F} \rightarrow \hat{E}$. By the continuity of $F$ and the definition of the topologies on $\hat{E}, \hat{F}$ we can find a sequence $\left(n_{k}\right)$ of indices such that $n_{k}<n_{k+1}$ and

$f\left(\left\{\left(x_{n}\right) \in \hat{\mathbb{H}}:\left\|\infty_{n}\right\| \leqslant 1\right.\right.$ for $\left.\left.n \leqslant n_{k}\right\}\right) \subset\left\{\left(y_{n}\right) \in \hat{\mathbb{B}}:\left\|y_{n}\right\| \leqslant k\right.$ for $\left.n \leqslant k\right\}$.

Now define $g_{k}: \tilde{P}_{n_{k}}(\hat{F}) \rightarrow l_{2}$ by $g_{k} \tilde{P}_{n_{k}}(x)=P_{k} f(x)$. This definition is unambiguous because $\tilde{P}_{n_{k}}$ is injective. First we check the continuity of $g_{k}$. Choose $\delta \leqslant 1$ such that

$$
\delta \leqslant \min \left\{\left\|\tilde{A_{n}} \ldots \tilde{A}_{n_{k-1}-1}\right\|: n<n_{k}\right\} .
$$

Then if $\left\|\tilde{P}_{n_{k}}(x)\right\| \leqslant \delta$, where $x=\left(x_{n}\right) \epsilon \hat{F}$, it follows that $\left\|\tilde{P}_{n} x\right\| \leqslant 1$ for $n \leqslant n_{k}$, so $\left\|P_{n} f(x)\right\| \leqslant k$ for $n \leqslant k$. In particular,

$$
\left\|g_{k} \tilde{P}_{n_{k}}(x)\right\|=\left\|P_{k} f(x)\right\| \leqslant k .
$$

Thus $g_{k}$ is continuous, so it may be extended to $f_{k}: l_{2} \rightarrow l_{2}$. We check the three conditions.

$$
\begin{aligned}
f_{k} \tilde{A}_{n_{k}} \ldots \tilde{A}_{n_{k+1}-1} \tilde{P}_{n_{k+1}} & =f_{k} \tilde{P}_{n_{k}}=g_{k} \tilde{P}_{n_{k}}=P_{k} f=A_{k} P_{k+1} f \\
& =A_{k} g_{k+1} \tilde{P}_{n_{k+1}}=A_{k} f_{k+1} \tilde{P}_{n_{k+1}} .
\end{aligned}
$$

Thus (i) holds on the range of $\tilde{P}_{n_{k+1}}$ which is dense by the corollary and hence (i) holds. For condition (ii) we observe that $f_{k} \tilde{P}_{n_{k}}=g_{k} \tilde{P}_{n_{k}}=P_{k} f$ which is injective. For (iii) let $y \in \hat{E}$ and take $x=f^{-1}(y) \in \hat{F}$. Then

$$
f_{k} \tilde{P}_{n_{k}}(x)=g_{k} \tilde{P}_{n_{k}}(x)=P_{k} f(x)=P_{k}(y),
$$

and the first part of the theorem has been completed. 
Next we observe that (ii) $\Rightarrow$ (ii)' and for the converse, we have for any $t$ that

$$
f_{1} P_{n_{1}}=A_{1} \ldots A_{k-1} f_{k} \tilde{P}_{n_{k}}
$$

and since $f_{1} P_{n_{1}}, A_{1} \ldots A_{k-1}$ are injective it follows that $f_{k} \tilde{P}_{n_{k}}$ is injective, Also it is clear that (iii) $\Rightarrow$ (iii)' and if we have (iii)' then for any $k$,

$$
A_{1} \ldots A_{k-1} f_{k} \tilde{P}_{k}(\hat{F})=f_{1} \tilde{P}_{n_{1}}(\hat{F}) \supset P_{1}(\dot{\hat{E}})=A_{1} \ldots A_{k-1} P_{k}(\hat{E}),
$$

so (iii)' follows from the injectivity of $A_{1} \ldots A_{k-1}$.

Finally, (iii)" follows from the observation that in any nuclear system $\left(A_{k}\right)$,we have

$$
P_{1}(\hat{E})=\bigcap_{k=1}^{\infty} A_{1} \ldots A_{k}\left(l_{2}\right)
$$

and the proof is completed.

We now apply Theorem 2 to nuclear spaces with bases, If $E$ is a nuclear Fréchet space whose topology is determined by norms and $E$ has. a Schauder basis then it follows (see [3], Chapt. 10) that $E$ is isomorphic to a nuclear sequence space. This means that $E$ is isomorphic to some $\hat{E}\left\{\left(D_{k}\right)\right\}$, where eaich $D_{k}: l_{2} \rightarrow l_{2}$ is a diagonal map. Furthermore each $D_{k}$ has only non-zero terms on its diagonal else it would fail to have dense range. Thus $\left(D_{k}\right)$ is an injective nuclear system. We see that Theorem 2 can be applied in several different ways depending on which form of the statement is used and on whether $\left(D_{k}\right)$ replaces $\left(A_{k}\right)$ or $\left(\tilde{A}_{k}\right)$. For convenience we state only one version.

CoRollart. Let $\left(A_{k}\right)$ be an injective nuclear system. Then $\hat{E}\left\{\left(A_{k}\right)\right\}$ has a Schauder basis if and only if there exist diagonal nuclear maps $D_{k}: l_{2} \rightarrow l_{2}$ and continuous linear maps $f_{k}: l_{2} \rightarrow l_{2}, k=1,2, \ldots$, such that

(i) $A_{k} f_{k+1}=f_{k} D_{k}, k=1,2, \ldots$,

(ii) $f_{1} D_{1}$ is injective,

(iii) $f_{1}\left(\bigcap_{k=1}^{\infty} D_{1} \ldots D_{k}\left(l_{2}\right)\right) \supset \bigcap_{k=1}^{\infty} A_{1} \ldots A_{k}\left(l_{2}\right)$.

The corollary permits us to establish the existence of Schauder bases for many examples of nuclear Fréchet spaces. We list a few here and save the details for a forthcoming paper which will be an extensive study of examples of nuclear systems.

$1^{0}$ If each $A_{k}$ is equal to a fixed $A: l_{2} \rightarrow l_{2}$ which is normal, then $\hat{E}$ has a basis.

$2^{\circ}$ If each $A_{k}$ is a weighted permutation of the usual basis vectors in $l_{2}$, then $\hat{E}$ has a basis.
$3^{0}$ If $\Omega$ is a sufficiently nice bounded open region in $\boldsymbol{R}^{n}$ and

$$
C_{0}^{\infty}(\bar{\Omega})=\left\{p \epsilon C^{\infty}(\Omega): D^{p} \varphi(\partial \Omega)=0 \text { for all } p\right\}
$$

and $C_{0}^{\infty}(\bar{\Omega})$ is given the topology of uniform convergence of each derivative, then $C_{0}^{\infty}(\bar{\Omega})$ is a nuclear Fréchet space with a Schauder basis.

Finally, we list some open questions which might have a bearing on the Schauder basis problem for nuclear Fréchet spaces.

$1^{0}$ Under what conditions is a sequence $\left(B_{k}\right)_{k}$ of operators on $l_{2}$ of the form $B_{l c}=A_{1} \ldots A_{k}$, where $\left(A_{k}\right)$ is some nuclear system.

$2^{\circ}$ Let $A: l_{2} \rightarrow l_{2}$ be a nuclear operator. Is the sequence space $\bigcap A^{k}\left(l_{2}\right)$ equal to a power series spacn (see [3]). Ist it even a "Stufenraum"? (see [2]).

$3^{0}$ Under what conditions on a nuclear system $\left(A_{k}\right)$ does there exist an operator $A$ on $l_{2}$ such that $\bigcap_{l_{i}} A_{l_{k}}\left(l_{2}\right)=A\left(l_{2}\right)$.

$4^{0}$ Is every nuclear system equivalent to an injective nuclear system?

Since every Fréchet nuclear space with a basis is the associated space of an injective nuclear system, a negative answer to this question would imply a negative answer to the basis question.

Added in proof. The details and computations in example $3^{\circ}$ are equivalent to those provided by H. Triebel, Math. Z. 90 (1965), p. 325-337. See also M. Zerner, C. R. Acad. Sc. Paris 268 (1969), p. 218-220.

\section{References}

[1] M. Do Wilde, Sur un type particular de limite inductive, Bull. Soc. Roy. Sci. Liège, $35^{\circ}$ Année, $17^{\circ} 9-10$ (1966), p. 545-551.

[2] G. Köthe, Topologische lineare Räume I, 1966.

[3] A. Pietsch, Nutclear : lokalknnvexe Räume, Berlin 1965. 